\title{
Modeling and Performance Analysis of a Flywheel Energy Storage System
}

\author{
Prince Owusu-Ansah, ${ }^{1,}$ Hu Yefa, ${ }^{1,}$ Philip Agyeman, ${ }^{1}$ Adam Misbawu ${ }^{2}$ \\ ${ }^{1}$ School of Mechanical and Electronic Engineering, Wuhan University of Technology, P.R China, \\ 430070 \\ ${ }^{2}$ School of Automation, Wuhan University of Technology, P.R China, 430070 \\ 라inosah1990@yahoo.co.uk hhuyefa@163.com ㄷillippo77@hotmail.com \\ ²misbaw6@yahoo.com,
}

Keywords: Rotor dynamic, Analysis, Rotor, Vibration, Deformation, Critical speed.

Abstract: This work discusses performance analyses of a flywheel energy storage system rotor using ansys. Design of a rotor based on 3D modeling and simulation is presented, the flywheel theory is also studied. A basic static structure and modal analyzes were carried out to evaluate the performance of the model rotor under these condition and the results discussed. Rotor analysis is performed in which a rotor model designed in solid works is imported into the ansys 14.5 environment for the analysis to be performed. The rotor model undergoes various forms of vibrations and deformation analysis using ansys 14.5 software and the results examined and discussed. Increasing rotor rotational speed above its safe critical limits influences the behavior of the natural frequency due to higher gyroscopic and moments of inertia which will eventually results in the non-performance of the rotor thereby causing total deformation to the rotor model during its operational cycle.

\section{Introduction}

A flywheel rotor is a component that rotates at a certain high velocity with a corresponding mass that will be required to store energy as it rotates (Genta 1985). The rotor which is also called the wheel, has been composed, in the past, by iron and other classical materials but new carbon fibers will make the wheel less heavy and with the ability to support much higher speeds. Rotating structure are pivotal components of high speed turbo machines used in modern day industrial machinery ranging from automobiles, high speed jet engines, marine propulsion as well as power station. (Dai et al. 2003) these rotating structures are commonly called "Rotors" and generally spin about an axis at high speed. (Bolund. et al. 2007). These rotors when made to rotate at high speed develop resonance, (Zhang \& Zhao 2004) this is a state at which harmonic loads are excited at their natural frequencies causing these rotors to vibrate excessively (Adams 2001). This vibration which usually are of larger amplitudes causes the rotors to bend and twist which leads to permanent failures during its cycle of operation. (Larsonneur 2009).

\section{Flywheel Theory}

Energy is stored in the rotor as kinetic energy, or specifically, a rotational energy

$\mathrm{E}=\frac{1}{2} I \omega^{2}$

The moment of inertia is a function of its shape and mass, given by equation,

$\mathrm{dJ}=\mathrm{dm} r^{2}$

$\mathrm{I}=\int x^{2} \mathrm{dm}\left[\mathrm{kg} / \mathrm{m}^{3}\right]$

Where $\mathrm{x}=$ distance from the rotational axis to the differential mass $d m x ; I=$ mass moment of inertia; and $\omega=$ angular velocity. 
Mass moment of inertia is obtained by the mass and geometry of the flywheel. For solid cylindrical disk, $\mathrm{I}$ is given as

$\mathrm{I}=\frac{1}{2} m r^{2}\left[\mathrm{~kg} / \mathrm{m}^{2}\right]$

Where $\mathrm{m}=$ mass and $\mathrm{r}$ the radius of the flywheel. Specific energy $\mathrm{E}_{\mathrm{k}} \mathrm{m}$ is obtained by dividing $\mathrm{E}_{\mathrm{K}}$ by the mass to give

$E_{k} m=\frac{1}{4} r^{2} \omega^{2}[J / J k g]$

If $E_{k}$, is multiplied through by the mass density $\rho$ of the flywheel, the energy density is obtained by

$E_{k} v=\frac{1}{4} \rho r^{2} \omega^{2}\left[J / m^{2}\right]$

\section{Structural Analysis}

Generally, considering the equation of multiple-degrees-of-freedom systems. (Jeng \& Gunter 2001)

$$
[\mathrm{M}]\{\ddot{D}\}+[\mathrm{C}]\{\dot{D}\}+[\mathrm{K}]\{\mathrm{D}\}=F
$$

Where $[\mathrm{D}]=$ displacements vector for a problem of $\mathrm{n}$ degree of freedom donated by [Di], $\mathrm{i}=1$, $2 \ldots \mathrm{n}$; $[\mathrm{F}]=$ external forces vector; $[\mathrm{M}]=$ mass matrix; $[\mathrm{C}]=$ damping matrix; and $[\mathrm{K}]$ is the stiffness matrix.

Equation (7) represents the governing equation of a transient structural simulation. The right hand side of the equation is the external force $[\mathrm{F}]$ and the first item of the left hand side of the equation, is inertia force, is damping force, and is the elastic force. (Lee 2012)

When analyzing the free vibration of a body there is no involvement of the external force $[\mathrm{F}]$. So, equation (7), becomes

$[\mathrm{M}]\{\ddot{D}\}+[\mathrm{C}]\{\dot{D}\}+[\mathrm{K}]\{\mathrm{D}\}=0$

The equation (8) is used for a problem having $n$ degrees of freedom. Our model has six degrees of freedom; it has at most six solutions of the fundamental natural frequency $(\omega)$. In a model analysis, we are interested in the natural frequencies and the relative shapes of the vibration modes. However, the damping effect can be neglected in the equation (8) which results in equation (9).

$[\mathrm{M}]\{\ddot{\mathrm{D}}\}+[\mathrm{K}]\{\dot{\mathrm{D}}\}=0$

Equation (9) is used in during modal analysis system to solve for the natural frequency for the six mode of freedom for the rotor model used in this analysis.

When using a stationery reference frame, the reference analysis system is attributed to the global coordinate system, which is a fixed one. In such analysis system, the gyroscopic moments due to nodal rotations are included in the damping matrix and the equation of motion becomes

$[M]\{\ddot{z}\}+([C]+[$ Cgyro $])\{\dot{z}\}+([K]+[H])\{z\}=\{f\}$

Where $[\mathrm{M}]=$ mass matrix; $[\mathrm{K}]=$ stiffness matrix; $[\mathrm{C}]=$ damping matrix; $\left[\mathrm{C}_{\text {gyro }}\right]=$ gyroscopic matrix; and $[\mathrm{B}]=$ rotating damping matrix

When using a rotating reference frame, the entire model rotates at the same rotational speed and the reference coordinate system also rotates along with the rotating parts (Kumar 2011). In rotating analysis system. (Schweitzer 1994).

The Coriolis terms are used in the equation of motion to describe rotational velocities and acceleration. So, the equation of motion for rotating reference frame is modified as

$([M]\{\ddot{z}\}+([C][C]+[$ Ccori $])\{\dot{z}\}+([K]-[K \operatorname{spin}])\{z\}=\{f\}(11)$

Where $[\mathrm{M}]=$ mass matrix; $[\mathrm{K}]=$ stiffness matrix; $[\mathrm{C}]=$ damping matrix; and $\left[\mathrm{C}_{\text {cori }}\right]=$ Coriolis mat 


\section{Design Structure}

The model was very easy to operate as well as easy to convert from the solidworks 13 to ansys 14.5 environment for the purpose of analysis Figure. 1 shows the rotor model designed in solid works 13 imported into ansys 14.5 is shown in Fig. 2.

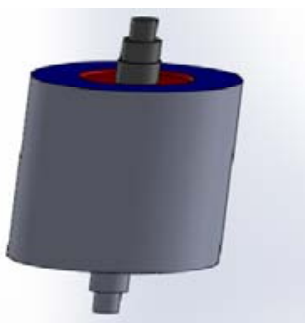

Fig. 1. Design Rotor Model

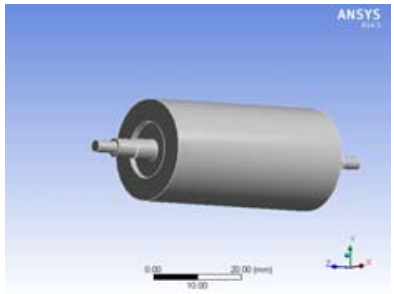

Fig. 2. Rotor in ansys

Table 1 Shows some of the parameters of the rotor and the permanent magnetic bearing.

\begin{tabular}{ll}
\hline Name of component model & Quantity \\
\hline Rotor mass & $10[\mathrm{~kg}]$ \\
Rotor diameter & $220[\mathrm{~mm}]$ \\
Designed energy storage & $300[\mathrm{wh}]$ \\
Maximum tip speed & $300[\mathrm{~m} / \mathrm{s}]$ \\
Maximum force for upper axial AMB & $400[\mathrm{~N}]$ \\
Maximum force for lower radial AMB & $550[\mathrm{~N}]$ \\
Working air gap & 0.2 \\
Bias current & $1.5[\mathrm{~A}]$ \\
\hline
\end{tabular}

\section{Finite Element Analysis}

Ansys 14.5 finite element analysis software was used for the calculation of the static structure analysis and the modal analysis during the course of the rotor model analysis.

To perform this process, a 3D model of the rotor was generated using solid works 2013 and subsequently imported to ANSYS 14.5. The material for the rotor model was chosen, and the force, torque and the boundary conditions were defined and selected.

\section{Modal Analysis}

It is very important to analyze the natural frequency of the rotor model to be able to forecast the vibration that occurs in these rotors. Modal analysis are performed in order to be able to predict the magnitudes of these vibrations as well as the structural vibration response of these high speed rotors, such as the natural frequencies and the mode shapes.

The meshing stage of the rotor performed is shown in Fig. 3 and the results of the six-order modal analysis are shown in Fig. 3 to Fig. 8 as well as the line frequency chart is also shown in Fig. 9.

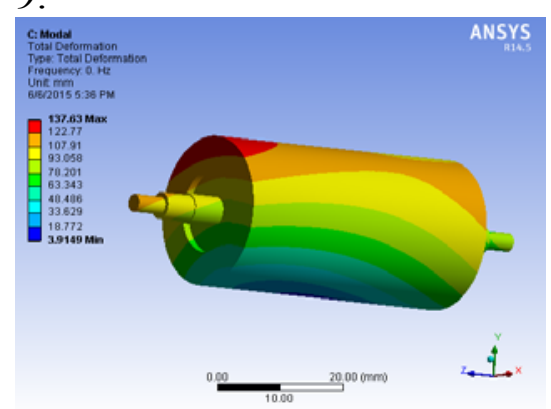

Fig. 3. Total deformation (Mode 1)

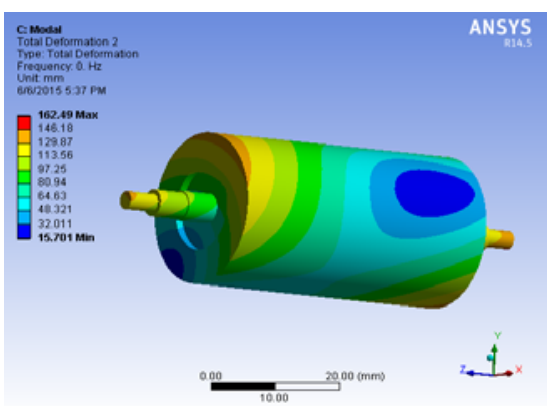

Fig. 4. Total deformation (Mode 2) 


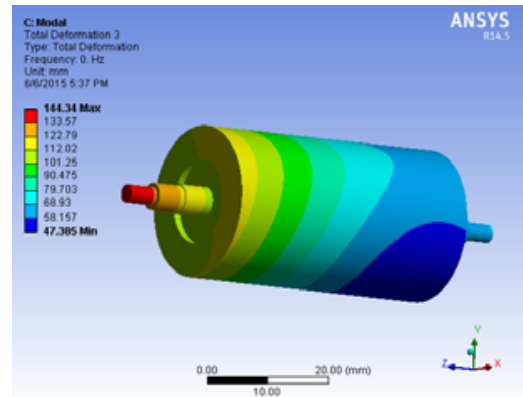

Fig. 5. Total deformation (Mode 3)

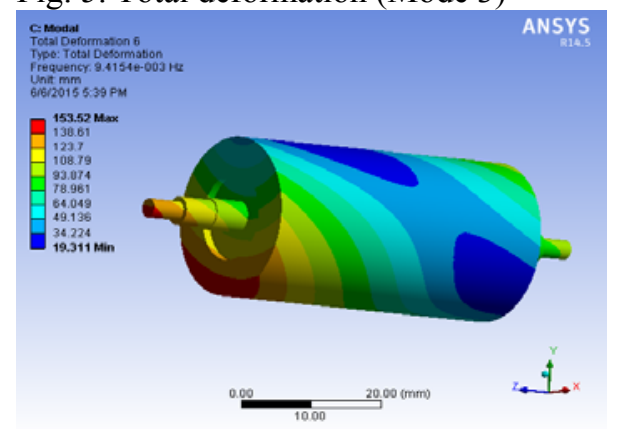

Fig. 7. Total deformation (Mode 5)

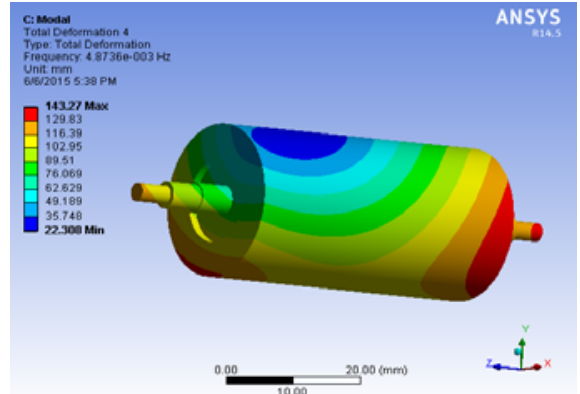

Fig. 6. Total deformation (Mode 4)

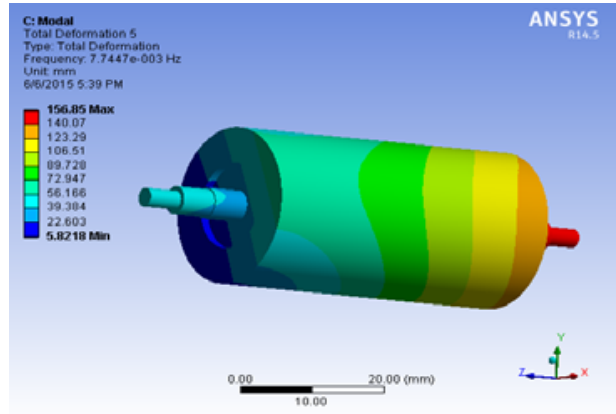

Fig. 8. Total deformation (Mode 6)

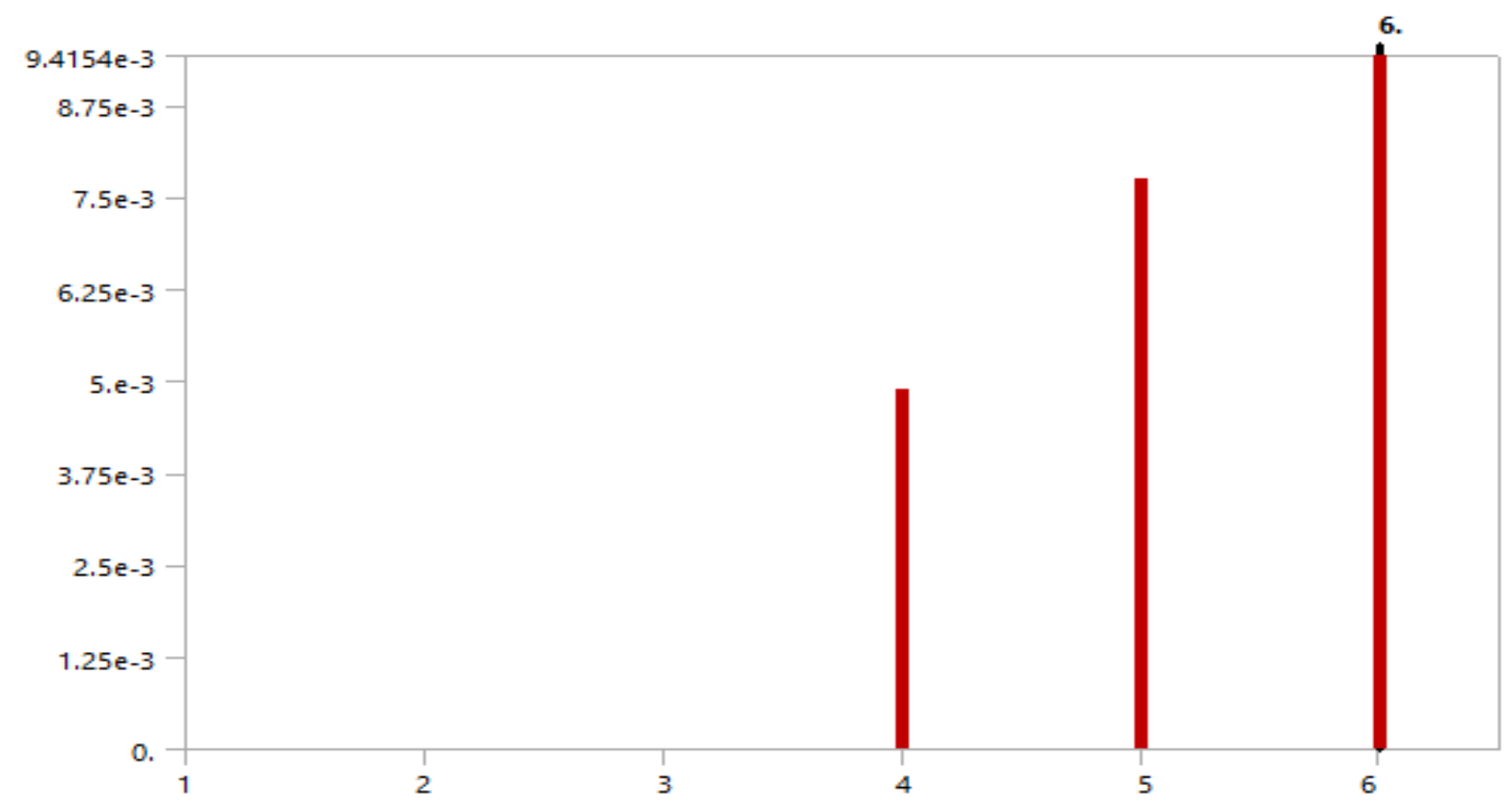

Fig. 9. Natural frequency rotor model.

It could be observed that for the first three order shown in Figs. 3 to 5 the natural frequency on the rotor was insignificant and as such no vibration was experienced on the rotor model, however, the last three modes. Mode 4 had a natural frequency of $4.8736 \mathrm{e}-003$ as shown in Fig 6. Mode 5 had a natural frequency of 7.7447e-003 as shown in Fig. 7, and the highest occurring at Fig. 8 at a frequency of $9.4154 \mathrm{e}-003 \mathrm{~Hz}$ with an input torque of $600 \mathrm{NM}$, it could be seen that as the speed increases the last three modes degrees begun to experience some sort of natural frequencies with the highest occurring in mode six and at this stage the rotor mode will begin to experience vibration.

Modal analysis in ansys software ensures that a forecast on the vibration and the natural frequencies of the model can be predicted. The calculated natural frequencies, the mode shapes, the 
damped frequency values are often used as a reference point for measurement, after which experimental modal analysis are usually performed for the free-free boundary conditions.

\section{Conclusion}

Performance analysis of a flywheel rotor using ansys through simulation has been studied. It has been shown through simulation that that ansys has the capability to handle more complex geometry models from solid works which can directly imported into ansys to be analyzed to give more accurate and definite results.

Figs. 6 to 10 shows that the mode shapes of the rotor increases due to the high centrifugal force which acts on the rotor model as the rotor speed increases thereby increasing the natural frequencies of the of the system which eventually increases the vibration modes of the rotor.

It can be concluded that increasing rotor rotational speed well above its safe critical limits influences the behavior of the natural frequency due to the higher gyroscopic which increases the vibration modes of the rotor model which eventual results in the non-performance hence causing total deformation to the rotor model during its operational cycle. It is therefore recommended that high speed flywheel rotors must be made to operate within the safe speed limit because operating these rotors above the safe critical speed will results in mechanical failures of the rotor components due to the high resulting centrifugal forces which are usually present during this stage of the rotor speed.

\section{Acknowledgments}

This work was supported by The Natural Science Foundation of China (NO.51275372) and Wuhan High-Tech Development Project Foundation (NO.201110921299) and The Fundamental Research Funds for the Central Universities (Wuhan University of Technology No.2012-IV-036).

\section{References}

[1] Adams, M. L., 2001 Rotating Machinery Vibration: From Analysis to Troubleshooting, Marcel Dekker.

[2] Bolund, B. et al., 2007. Renewable and Sustainable Energy Reviews.pp. 235-258.

[3] Dai, X.J, Wei, H.G. and Shen, Z.P 2003. Dynamic design and experimental study of the rotor bearing system of a flywheel energy storage system, Chinese Journal of Mechanical Engineering, 39(4) 97-101.

[4] Genta, G., 1985. Kinetic energy storage Theory and practice of advanced flywheel systemsButterworth, page 58, ISBN 0-408-01396-6.

[5] Jeng, W., and Gunter, E. 2001. Introduction to Dynamics of Rotor-Bearing Systems, Eigen Technologies.

[6] Kumar, S. 2011. Rotor Dynamic Analysis using ANSYS: Proceedings of the IUTAM symposium on Emerging trends in rotor Dynamic: Edited by K Gupta, Spring New York.

[7] Larsonneur, R., 2009. Control of the Rigid Rotor in AMBs”. In Magnetic Bearings - Theory, Design, and Application to Rotating Machinery,

[8] G. Schweitzer and E. Maslen, eds. Springer Verlag, Berlin, Chapter 8, pp. 170-228.

[9] Lee, H.-H., 2012. Finite Element Simulations with ANSYS Workbench 14.SDC publications Schweitzer,

[10]G., and Bleuler, H. 1994. A Traxle, Basic design and theory of magnetic bearings, Active Magnetic Bearings basics properties and applications, Verlagm der Fachvereine (vdf). 
[11]Vance, J., Zeidan F. and Murphy, B. 2010. Machinery Vibration and Rotor dynamics, John Wiley \& sons, Inc.,

[12]Zhang, K., and Zhao, H. 2004. Research on flywheel suspended by AMB, s with significant gyroscopic effect, Journal of Chinese Mechanical Engineering, 2004, 17(1):63 